\title{
Magnetization precession due to a spin polarized current in a thin nanoelement: numerical simulation study
}

\author{
D.V. Berkov, N.L. Gorn \\ Innovent e.V., Prüessingstr. 27B, D-07745, Jena, Germany
}

(Dated: June 24, 2021)

\begin{abstract}
In this paper a detailed numerical study (in frames of the Slonczewski formalism) of magnetization oscillations driven by a spin-polarized current through a thin elliptical nanoelement is presented. We show that a sophisticated micromagnetic model, where a polycrystalline structure of a nanoelement is taken into account, can explain qualitatively all most important features of the magnetization oscillation spectra recently observed experimentally (S.I. Kiselev et al., Nature, 425, 380 (2003)), namely: existence of several equidistant spectral bands, sharp onset and abrupt disappearance of magnetization oscillations with increasing current, absence of the out-of-plane regime predicted by a macrospin model and the relation between frequencies of so called small-angle and quasichaotic oscillations. However, a quantitative agreement with experimental results (especially concerning the frequency of quasichaotic oscillations) could not be achieved in the region of reasonable parameter values, indicating that further model refinement is necessary for a complete understanding of the spin-driven magnetization precession even in this relatively simple experimental situation.

PACS numbers: 85.75.-d, 75.75.+a, 75.40.Gb, 75.40.Mg
\end{abstract}

\section{INTRODUCTION}

Magnetic excitations and magnetization switching in nanostructures induced by a spin-polarized current (SPC), first predicted theoretically ${ }^{\underline{1}}$ and soon discovered experimentally ${ }^{2}$ are now one of the most intensively studied topics in solid state magnetism due to their importance both from the fundamental point of view and for numerous possible technical applications in, e.g., microwave generators and MRAM cells (see recent reviews ${ }^{3}$ ). In the past decade a substantial progress has been achieved by the elaboration of analytical theories explaining the spin-transfer driven magnetization dynamics and by refining experimental techniques in order to enable quantitative experimental studies of these phenomena.

Analytical models employing the scattering matrix formalism ${ }^{4}$ and taking into account spin-dependent reflection and transmission of electrons, precession of electron spins and the structure of Fermi surfaces for concrete materials s. $^{5.6}$ have been developed which allow the calculations of the spin-transfer torque arising due to the current crossing the normal metal-ferromagnet interface. The derivation of the density operator corresponding to the normal metal spacer (N) in a FM/N/FM structure combined with a two-current (for spin-up and spin-down electrons) model enabled the evaluation of a spin torque in a complete trilayer structure ${ }^{7.8}$ which was successfully tested and extended using the Boltzmann equation formalism ${ }^{9}$. This way an asymmetric dependence of the spin torque on the angle between the two FM layers could be derived ${ }^{6.7 .8}$ whereby the parameters governing this asymmetry depend on the conductivities and geometry of the multilayer device under study. A large effort has also been made to establish the relation between the spin-transfer phenomena, interlayer exchange coupling and Gilbert damping in multilayer structures (see Ref. 10 11,13 and citations therein).

At the same time first high quality experimental results were obtained by studying magnetization oscillations induced by a spin-polarized current in various systems. Magnetization dynamics in nanopillars was investigated in Ref. 14 15 16 17 18. Detailed studies of the steady-state magnetization oscillations were reported also for the pointcontact geometry ${ }^{19,20,21}$. In these papers several different precession modes were identified and the dependencies of the precession frequency and microwave oscillation power on the external field and dc current strength were investigated.

Quantitative comparison between theory and experiment remains, however, a delicate issue due to fairly complicated remagnetization processes involved. Experimentally accessible multilayered nanostructures have thicknesses in the range of several nanometers and the lateral sizes about $100 \mathrm{~nm}$ and larger which is far above the critical size for the single-domain behaviour. For this reason one expects the formation of relatively complex domain structures during the magnetization switching, especially taking into account that for most equilibrium magnetization states the spin-induced torque has different directions for different parts of the nanoelement (see, e.g., the discussion in Ref. 22). Indirect evidences for such a domain-mediated switching can be found already in first semiquantitative experimental studies ${ }^{23}$, where a quasistatic switching of a nanoelement by changing the external field or the electric current strength was investigated. Recent experiments have confirmed that a macrospin (single-domain) approximation can not explain several important observations concerning the magnetization dynamics in nanopillars and point contacts 14.18 .20 , although the macrospin model was able to predict some of its qualitative aspects like the existence of a steady-state precession regime by itself (for the most detailed study in frames of a macrospin approach see Ref. 
24).

For this reason the full finite-element micromagnetic modelling is required in order to understand whether the discrepancies between theoretical predictions and experimental observations are due to a poor theoretical understanding of a basic physics involved or because the formation of complicated domain structure significantly alters the experimentally measurable system properties. Until the present time very few such studies have been carried out. In the pioneering paper $^{22}$ devoted to this subject the formation of complicated magnetization states already in a nanoelement with lateral sizes as small as $125 \times 125 \mathrm{~nm}$ was found. In the latter detailed study $\underline{25}$ of the magnetization dynamics of a $64 \times 64 \mathrm{~nm}$ square with the thickness $2.5 \mathrm{~nm}$ it was predicted that the behaviour of this element is nearly single-domain. However, the results of Ref. 25 turned out to be at least partially incorrect for the parameters of magnetic materials typically used experimentally: it was shown ${ }^{26.27}$ that for a nanoelement with this thickness significant deviations from a single-domain behaviour appear already for lateral sizes $b \approx 30 \mathrm{~nm}$ and a completely chaotic magnetization pattern was found for $b \geq 60 \mathrm{~nm}$, which clearly emphasizes the importance of full-scale micromagnetic simulations.

Up to our knowledge, systematic micromagnetic studies concerning the spin-injection driven magnetization dynamics have been performed only for a SPC induced noise in spin-valves 28 . In addition, in several short reports ${ }^{29.30}$ some qualitative aspects of the magnetization dynamics of elliptical nanoelements (concerning mainly the transition from a homogeneous to a non-coherent magnetization precession) were discussed.

In this paper we present a systematic study of the magnetization dynamics of an elliptical nanoelement under the influence of a spin-polarized current. The paper is organized as follows: In Sec. [II we outline our simulation methodology, paying special attention to the justification of our choice of the simulation parameters. In Sec. III we present our simulation results, starting with a 'minimal' micromagnetic model and carrying on with adding the effects of a polycrystalline structure and thermal fluctuations. In the same section we analyze also the influence of the exchange stiffness constant on the magnetization dynamics, keeping in mind that among various magnetic parameters of a ferromagnetic material the experimental determination of this constant is the most difficult task. In Sec. IV we compare our results first with numerical simulations of other groups and then with the experimental studies of the microwave oscillations in such a nanoelement. At the end of this last Section we discuss how the factors which were not included in our model could affect the magnetization dynamics of the system under study.

\section{NUMERICAL SIMULATIONS: METHODOLOGY AND CHOICE OF MATERIAL PARAMETERS}

Numerical simulations were carried out using our package MicroMagus 31 , whereby the spin injection was included in form of the Slonczewski torque $\Gamma=\left(a_{J} / M_{S}\right) \cdot[\mathbf{M} \times[\mathbf{M} \times \mathbf{S}]]$ ( $\mathbf{S}$ is the spin polarization direction of the current through a nanoelement). The time evolution of the magnetization configuration was obtained by integrating the LandauLifshitz-Gilbert equation of motion for the system magnetization using the modified Bulirsch-Stoer algorithm. This method includes an adaptive step size control, what is especially important when simulating dynamics of a strongly non-homogeneous magnetization configuration.

Keeping in mind the intended comparison of our simulation results with those obtained experimentally, we have made an effort to choose the system parameters as close as possible to their values for experimentally studied nanoelements. Among these experimental studies we have selected the publication of the Cornell group $\stackrel{14}{ }$, where ones of the qualitatively most interesting results on $\mathrm{Co} / \mathrm{Cu} / \mathrm{Co}$ system were reported.

An elliptical element with the geometry corresponding to that used in Ref. 14, i.e., with lateral sizes $130 \times 70 \mathrm{~nm}$ and thickness $d=3 \mathrm{~nm}$ was simulated. The element was discretized in plane using the mesh size $2.5 \times 2.5 \mathrm{~nm}$ (we have checked that further mesh refinement did not lead to any noticeable changes in the simulation results).

The choice of the magnetic material parameters requires special justification.

The saturation magnetization of thin Co layers, as it is well known, may be different from that of the bulk material. For this reason we have used the saturation magnetization $4 \pi M_{S}=10 \mathrm{kG}$ as measured in Ref. 14 on a $\mathrm{Co} / \mathrm{Cu}$ multilayer system where each Co layer was $3 \mathrm{~nm}$ thick (see below also our analysis concerning the influence of the saturation magnetization value on the final results).

The value of the exchange stiffness constant $A$ for a bulk Co and thin Co film is also a subject of controversial discussions. Some earlier measurements have lead to the values in the range $A=(1-2) \times 10^{-6} \mathrm{erg} / \mathrm{cm}$ (see Ref. 32 and citations in Ref. 33, pp. 368-381). In latter experiments ${ }^{34}$ performed using the inelastic neutron scattering on bulk Co crystals and recent measurements $\frac{35}{3}$ carried out on thin Co films using the elastic small-angle neutron scattering significantly higher values $A=(2.8-3.1) \times 10^{-6} \mathrm{erg} / \mathrm{cm}$ have been found. For this reason we have chosen the value $A=3.0 \cdot 10^{-6} \mathrm{erg} / \mathrm{cm}$ as a basis and have also studied how the smaller values of $A$ would affect the results.

The treatment of the Gilbert damping (represented in the standard version of the LLG-equation by the double vector product $\left[\mathbf{M} \times\left[\mathbf{M} \times \mathbf{H}^{\text {eff }}\right]\right]$ multiplied by a constant factor $\left.\lambda\right)$ is at present a subject of an intensive research and is also closely related to the problem of a correct choice of the spin torque term. First, even in the absence of an externally 
driven spin-polarized current the value of the dissipation constant in thin film systems may substantially deviate from its value for the corresponding bulk material (see a short survey of experimental data collected in Ref. 10). This effect is usually attributed to a spin transfer (and subsequent relaxation) from a ferromagnet into a normal metal layer ${ }^{10}$ and/or to a similar process leading to a "dynamic exchange coupling" between two FM layers 11 . In addition, it was shown ${ }^{12}$ that due to the dependence of the spin pumping and the spin accumulation on the angle between the magnetization of FM layers in a FM-N-FM systems the damping depends on the instantaneous magnetization configuration of FM layers.

The same mechanisms are responsible for a non-trivial dependence of the magnitude of the spin torque $\boldsymbol{\Gamma}$ on the angle $\theta$ between the FM layer magnetizations (the trivial part of this dependence, $\Gamma \sim \sin \theta$, is contained in the double vector product $[\mathbf{M} \times[\mathbf{M} \times \mathbf{S}]])$. Various theoretical approaches ${ }^{7.9 .36}$ have led to the conclusion that in a symmetric spin valve the functional dependence of the spin torque on $\theta$ can be cast into the form $\Gamma(\theta) \sim P \Lambda^{2} \sin \theta /\left[\left(\Lambda^{2}+1\right)+\left(\Lambda^{2}-1\right) \cos \theta\right]$, where $P$ is proportional to the difference between the resistances for the electrons with opposite spin directions and $\Lambda$ may be expressed as a function of a total resistance and conductance of a non-magnetic metallic spacer. For a non-symmetric spin valves more complicated formulae were derived ${ }^{9}$.

Both topics discussed above - (i) dependence of the dissipation parameter $\lambda$ on the average and local characteristics of a multilayer and (ii) a complicated dependence of a spin torque magnitude on the angle between the magnetization vectors in adjacent layers - can and should in principle be incorporated into micromagnetic simulations. However, the study of the influence of these factors on the magnetization dynamics is for obvious reasons a separate and difficult problem. In our opinion, this problem should be addressed after the magnetization dynamics driven by a spin-polarized current is sufficiently well understood for the model where both the damping parameter and the coefficient before the Slonczewski torque term are assumed to be constant. As we shall see below, this is still not the case if one goes beyound the macrospin approximation, i.e., when the complicated magnetization structures arising in nanoelements under realistic conditions are taken into account. For this reason we have decided to put both $\lambda=$ Const and $a_{J}=$ Const (so that $\Gamma(\theta) \sim \sin \theta$ ) in order to find out (i) which qualitative features of the experimentally observed steady state precession and switching of magnetic nanoelements can be explained within this simplified treatment of the spin-transfer induced torque and (ii) whether this model can provide quantitative agreement with experimental data for reasonable parameter values. In future, the comparison of our results presented here with simulations employing more sophisticated spin torque models will allow the unambiguous identification of dynamic phenomena arising due to the abovementioned non-trivial properties of the Gilbert damping and the complicated $\theta$-dependence of the spin torque magnitude.

Within the approach outlined above we are left with two problems: how to choose the value of the Gilbert damping $\lambda$ and the proportionality coefficient between the experimentally measured current values $I$ and the Slonczewski term magnitude $a_{J}$ for our system. We are not aware of any independent measurements of $\lambda$ for the experimental situation similar to that studied in Ref. 14, which are reliable enough to be used as a simulation input. Hence we have adopted the following strategy to choose this parameter. First, it is well known from general considerations and confirmed by numerical simulations ${ }^{28}$ that the current threshold $I_{\min }$ for the onset of the steady state magnetization oscillations growth linearly with $\lambda$. At the same time, we have found that the critical current value $I_{\max }$ for which the oscillations disappear is nearly independent on the damping. For this reason we have chosen the damping which enabled us to reproduce a ratio of the maximal and minimal currents $I_{\max } / I_{\min }$ for which a significant microwave power was observed experimentally ${ }^{14}$. This procedure lead to the value $\lambda \approx 0.04$. This damping is significantly higher than the usually reported values of $\lambda$ in $\operatorname{Co}(\lambda \sim 0.01-0.02)$ which might indicate that the effects discussed above lead (on average) to a substantial enhancement of the damping in the system under study.

Establishing of the relation between the total current $I$ (or the current density $j$ ) and the spin torque magnitude $a_{J}$ is required for the calculation of the current induced magnetic field (Oersted field). Following the discussion above, we did not try to calculate the corresponding proportionality coefficient using various available theoretical models. Instead, we have again used for this purpose the value of the current threshold $I_{\min }$ reported in Ref. 14. Namely, we have calculated the Oersted field for the critical current value $I_{\min }$ (which is $\approx 2 \mathrm{~mA}$ for $H_{\text {ext }}=2 \mathrm{kOe}$ in Ref. 14) corresponding to the onset of steady state oscillations. Afterwards, keeping the Oersted field constant, we have increased the value of $a_{J}$ until the steady state precession emerged thus obtaining the critical value of the spin torque $a_{J}^{\mathrm{cr}}$. The ratio between $I_{\min }$ and $a_{J}^{\mathrm{cr}}$ was then used as a constant proportionality coefficient between $a_{J}$ and $I$ (we need the latter to calculate the Oersted field for the known geometry) when we increased the spin torque value $a_{J}$ in our studies.

We have chosen the coordinate system with $0 x z$ plane coinciding with the element plane and $0 x$ axis parallel to the long ellipse axis. All simulation results presented here were obtained for the external field $\mathbf{H}_{0}$ with components $H_{0 x}=2000 \mathrm{Oe}, H_{0 y}=20 \mathrm{Oe}, H_{0 z}=100 \mathrm{Oe}$. The $H_{0 x}$-value corresponds to that used for several measurement series reported in Ref. 14, the small in-plane deviation $H_{0 z}$ was introduced to mimic a corresponding small angle between the long ellipse axis and the external field ${ }^{14}$, and the very small out-of-plane component $H_{0 y}$ was introduced to remove an artificial numerical degeneracy present when the field is applied exactly in the layer plane. The spin polarization 
vector $\mathbf{S}$ was chosen to be antiparallel to the external field thus assuming that the magnetization direction of the polarizing (lower) layer coincides with the external field (the upper layer magnetization dynamics is driven by the electrons reflected from the polarizing layer so that their average spin polarization is opposite to that of the lower layer).

For a direct comparison of simulation results with experimental data we need to calculate the spectral power of the resistance oscillations. To do this, we make an assumption that in the CPP-geometry (current perpendicular to plane) the resistance variation due to the magnetization dynamics for the current flowing through the $i$-th discretization cells of the free (magnetization direction $\mathbf{m}_{i}^{\text {free})}$ ) and fixed $\left(\mathbf{m}_{i}^{\text {fix }}\right)$ layers can be written as $\Delta R_{i}=\Delta R_{\max }\left(1-\cos \theta_{\mathrm{i}}\right) / 2=\Delta \mathrm{R}_{\max }\left(1-\left(\mathbf{m}_{\mathrm{i}}^{\text {free }} \mathbf{m}_{\mathrm{i}}^{\mathrm{fix}}\right)\right) / 2$. We note in passing that at present more complicate dependencies of the magnetoresistance on the angle between the adjacent layer magnetizations are discussed (in addition to the papers mentioned above, see, e.g., Ref. 8). However, at this stage of our research where we have to use the angular-independent spin torque magnitude $a_{J}$, we prefer to use the standard cos-like dependence written above thus treating both phenomena (magnetoresistance and spin torque) in a self-consistent manner.

For the device under consideration the variation of the resistance due to the magnetization precession is much lower than its value in the absence of magnetoresistive effects. Using this simplifying assumption, the total timedependent resistance variation of our discretized micromagnetic model treated as a system of $N_{c}$ parallel resistors can be expressed as a function of the fixed layer magnetization direction $\mathbf{m}^{\text {fix }}$ (this layer is assumed to be homogeneously magnetized) and the average magnetization direction $\left\langle\mathbf{m}^{\text {free }}\right\rangle$ of the free layer as

$$
\Delta R_{\text {tot }}(t)=\frac{\Delta R_{\max }}{2 N_{c}}\left\{1-\left(\mathbf{m}^{\text {fix }} \cdot\left\langle\mathbf{m}^{\text {free }}\right\rangle\right)\right\}
$$

Taking into account that the in-plane magnetization direction of the fixed layer for the geometry under study coincides with in-plane external field direction (given by the unit vector $\mathbf{e}^{h}=\mathbf{H}_{0} / H_{0}$ ) and that the out-of-plane component of $\mathbf{m}^{\text {fix }}$ is vanishingly small, we finally obtain the formula

$$
\Delta R_{\mathrm{tot}}(t)=\frac{\Delta R_{\max }}{2 N_{c}}\left\{1-\left(e_{x}^{h}\left\langle m_{x}^{\text {free }}\right\rangle+e_{z}^{h}\left\langle m_{z}^{\text {free }}\right\rangle\right)\right\}
$$

which enables a direct calculation of the magnetoresistance oscillation spectrum from the known time dependencies of the average in-plane magnetization projections of the free layer.

For further references we also note that the frequency of the homogeneous FMR-mode of the extended thin film with magnetic parameters and under external conditions given above would be $f_{\mathrm{FMR}}^{(0)}=(\gamma / 2 \pi) \cdot\left(H_{0}\left(H_{0}+4 \pi M_{S}\right)\right)^{1 / 2} \approx 13.7$ $\mathrm{GHz}$.

\section{NUMERICAL SIMULATIONS: RESULTS AND DISCUSSION}

\section{A. Magnetization dynamics for $T=0$ : a 'minimal' micromagnetic model with the reference parameter set}

We begin with the description of the steady state precession without thermal noise. In this case there exist for a fixed external field and increasing current (increasing $a_{J}$ in our formalism) a sharp transition from a stable magnetization state (time-independent magnetization configuration) to a steady-state regime with regular oscillations. In this subsection we consider a 'minimal' micromagnetic model, where the polycrystalline structure of a nanoelement is neglected, so that no random magnetic anisotropy of crystal grains is taken into account.

We start the analysis of our simulation results from the regular oscillation regime, which is characterised by a nearly homogeneous magnetization configuration during the precession. For the nanoelement parameters $\left(4 \pi M_{S}=10 \mathrm{kG}\right.$, $A=3.0 \cdot 10^{-6} \mathrm{erg} / \mathrm{cm}$ and $\left.\lambda=0.04\right)$ and the external field $\left(\mathbf{H}_{\mathbf{0}}=(2000,20,100)\right.$ Oe $)$ given above, the current threshold for the oscillation onset in our "minimal" model was found to be $a_{J}^{\text {cr }}=0.3310(5)$. When $a_{J}$ is increased above this value, a very fast growth of the oscillation amplitude was observed. In particular, for $a_{J}=0.333$ the oscillation amplitude of the average $z$-projection of the element magnetization $m_{z}^{\text {av }}$ (the in-plane projection along the short ellipse axis, nearly perpendicular to the external field) was already $\Delta_{z} \approx 0.7$ (see Fig. 10). The regular oscillation regime exists in a extremely narrow $a_{J}$-region $0.331 \leq a_{J} \leq 0.355$. In this regime the spectrum of $m_{z}^{\text {av }}$-oscillations contains only a single very narrow peak which frequency is $f_{1} \approx 10.37 \mathrm{GHz}$ for $a_{J}=0.333$ and decreases down to $f_{1} \approx 9.14 \mathrm{GHz}$ for $a_{J}=0.350$. We point out that already for $a_{J}=0.333$ the steady-state oscillation frequency is well below the frequency of the homogeneous FMR-mode $f_{\mathrm{FMR}}^{(0)}$, although the distribution of the oscillation power is almost perfectly homogeneous (Fig. 10, left panel). The reason is that even for this 
$a_{J}$-value - which is very close to the oscillation onset threshold $a_{J}^{\mathrm{cr}}$ - the oscillation amplitude is so large that the formula $f_{\mathrm{FMR}}^{(0)}=(\gamma / 2 \pi) \cdot\left(H_{0}\left(H_{0}+4 \pi M_{S}\right)\right)^{1 / 2}$ used to calculate $f_{\mathrm{FMR}}^{(0)}($ where small deviations of magnetization from its equilibrium orientation are assumed) is not applicable.

As mentioned above, the regular oscillation regime with a well defined limiting cycles for the average system magnetization (Fig. 1b, right panel) persists up to $a_{J}^{\text {ch }} \approx 0.355$. Further small increment of the current strength leads to an abrupt transition to a quasichaotic magnetization motion (whether it is truly chaotic in the sense of non-linear dynamics should be investigated separately; see also our brief discussion in Ref. 26). An example of the magnetization trajectories in this quasichaotic regime is given in Fig. 15.

For currents not much larger than the transition value $a_{J}^{\text {ch }}$ the oscillation spectra of the average magnetization components still exhibit a well defined structure with several peaks corresponding to several oscillation eigenmodes with different spatial distribution of the oscillation power. An example of the such a spectrum with spatial maps attributed to different spectral peaks is given in Fig. 1: (left panel). In-plane spatial distributions of the oscillation power were calculated from the time-dependent trajectories of the discretization cell moments as described in Ref. 27. The detailed analysis of these spatial structures is beyound the scope of this paper. Here we would like only to mention that the asymmetry of the spatial distributions for the second and third modes is due to the presence of the Oersted field. Although this field is much smaller than the external field, its influence for this particular case is visible due to a relatively large amplitude of the magnetization oscillation.

Further increment of the current strength $\left(a_{J}\right.$ value) results in the gradual decline of the oscillation power for both in-plane magnetization components, accompanied by the decrease of the oscillation frequency as a function of $a_{J}$. This behaviour is demonstrated in Fig. 2] where we present the maps of $m_{x}^{\text {av }}$ - and $m_{z}^{\text {av }}$-oscillation power as functions of the frequency (vertical axis) and $a_{J}$ (horizontal axis) together with the magnetoresistance oscillation power computed according to Eq. (2). Characteristic for this dynamical regime is a multi-domain magnetization configuration whereby the average domain size decreases with increasing $a_{J}$. Because the average magnetization is still directed along the external field, a formation of such a chaotic domain structure with smaller and smaller domains leads on average to the increase of the stray field in the opposite direction, which might be one of the reasons why the oscillation frequency decreases with $a_{J}$.

It is important to note, that in contrast to $m_{z}^{\text {av }}$, the oscillation power of the $m_{x}^{\text {av }}$-component (parallel to the long ellipse axis) is concentrated in two frequency regions. First, there is a contribution from fast $m_{x}^{\text {av }}$ oscillations at the frequency approximately twice as high as for $m_{z}^{\text {av }}$ oscillations. The reason for this relation between $m_{x}$ and $m_{z}$ oscillation frequencies is that for the motion type under study - the magnetization is precessing around (approximately) the $0 x$-direction - the $m_{x}$-component moves back and forth twice during one complete oscillation cycle (see, e.g., right graphs in Fig. 1b and c). Second, there exist a substantial low-frequency contribution to the oscillation power (which growth with increasing $a_{j}$ ) due to slow variations of $m_{x}^{\text {av }}(t)$ which are typical for a quasichaotic motion. Analogous behaviour of the longitudinal magnetization component in similar situations was observed in Ref. 26]282930 (see also the discussion below).

Increasing $a_{J}$ above the value $a_{J} \approx 1.4$ leads to the disappearance of the chaotic regime and to the establishing of a so-called 'out-of-plane' precession mode. The magnetization trajectory in this mode is an ellipse with the plane slightly tilted with respect to the nanoelement plane. The oscillation frequency abruptly increases and is now for obvious reasons the same for $m_{x}^{\text {av }}$ and $m_{z}^{\text {av }}$-components (see regions marked by circles in Fig. 2). The magnetization is precessing nearly coherent in this mode, what can be seen from a large absolute value of the out-of-plane magnetization projection: $\left(m_{y}^{a v} \approx 0.8-0.9\right)$. The absolute value of the oscillation power in this regime is at least an order of magnitude smaller than at the beginning of the quasichaotic motion due a relatively small precession amplitude.

Comparison of these results with experimental data and numerical simulations of other groups will be made in Section IV

\section{B. Magnetization dynamics for $T=0$ : influence of the exchange stiffness value}

Taking into account a relatively broad region of the exchange stiffness constants reported for bulk Co and thin Co films (see discussion at the beginning of this Section), we have studied how the decrease of $A$ would affect the magnetization dynamics. From general considerations, we expect the magnetization configuration to become 'softer' for smaller exchange stiffness, so that the influence of a strongly inhomogeneous self-demagnetizing field of a flat elliptical nanoelement should lead to an equilibrium magnetization configuration which is less collinear compared to a system with larger $A$. This, in turn, should result in a broader distribution of the resonance fields for different regions of a nanoelement (different discretization cells in our micromagnetic model), thus leading to a slower increase of the magnetization oscillation amplitude with increasing $a_{J}$ (above the oscillation threshold $a_{J}^{\text {cr }}$ ). In addition, for smaller exchange the transition from a regular to a quasichaotic oscillation regime is expected to occur earlier (for smaller $\left.a_{J}\right)$. 
All these features were indeed observed in our simulations, whereby it has turned out that from the quantitative point of view the nanoelement with the geometry under study is quite sensitive already to moderate decrease of $A$. In Fig. 3 we display an example of the transition from a regular to a quasichaotic behaviour for the nanoelement with parameters identical to those given in the preceding subsection, but with $A=2.0 \cdot 10^{-6} \mathrm{erg} / \mathrm{cm}$. The oscillation threshold $a_{J}^{\text {cr }}=0.3290(5)$ is almost the same as for the system with the reference parameter set. However, the spectral power of magnetization oscillations in a regular regime is substantially smaller than for a 'reference' system. In particular, when $a_{J}$ exceeds the oscillation threshold value by $\Delta a_{J}=0.0015\left(a_{J}=0.331\right.$, see corresponding spectrum in Fig. $3 \mathrm{k}$ and trajectories in Fig. [3 $)$ ), the oscillation amplitude is significantly smaller than for the same situation for a 'reference' system (Fig. [1). For this reason the oscillation frequency here $(f \approx 12.45 \mathrm{GHz})$ is much closer to $f_{\mathrm{FMR}}^{(0)}$ than for the peak shown in Fig. 1b.

The transition to a quasichaotic motion occurs, due to the reason explained above, for a somewhat smaller current value $a_{J}^{\text {ch }}=0.338(1)$. Interestingly, the non-trivial mode structure appears for this system already before the transition (see spectra for $a_{J}=0.334$ and $a_{J}=0.336$ in Fig. 3 3 ). In addition, for the system with smaller $A$ only the first and third mode from of the eigenmode set identified for $A=3.0 \cdot 10^{-6} \mathrm{erg} / \mathrm{cm}$ are visible in the power spectrum of magnetization oscillations just above the transition to chaos. The analysis of trajectories for individual discretization cells reveals that the second mode (with the intermediate frequency) is still present. However, the spatial structure of this mode for this smaller exchange value is nearly symmetric (compare oscillation power maps in Fig. 11; and 3r), and the mode does not manifest itself in the spectrum of the average magnetization dynamics, because the magnetization in the left and right mode localization regions oscillates in opposite phases.

Analysing the influence of exchange stiffness value on the overall spectral picture in the chaotic regime, we can see that the decrease of $A$ increases the upper boundary of the current region where the quasichaotic regime exists (compare Fig. $5 \mathrm{~F}$ and $5 \mathrm{~b}$ ). In addition, it can be seen that the decrease of $A$ down to $A=2.0 \cdot 10^{-6} \mathrm{erg} / \mathrm{cm}$ leads to a nearly complete disappearance of the 'out-of-plane' precession region. This means that for large spin torque values this exchange constant is not high enough to ensure the collinear magnetization structure necessary for the very existence of the 'out-of-plane' precession mode.

\section{Magnetization dynamics for $T=0$ : influence of the random crystal grain anisotropy}

It is well known that thin sputtered Co films have a polycrystalline structure which in the simplest case can be treated as absolutely random and characterised by a single parameter - the average grain size $\langle D\rangle$. In all the simulations which results are shown below we have used $\langle D\rangle=10 \mathrm{~nm}$. To study a nanoelement with such a random polycrystalline structure we have applied a standard procedure included into the MicroMagus 31 package. The procedure starts with the random placement of 'growth centers' within the simulated nanoelement, whereby the number of growth centers is determined from the nanoelement size and the average grain size $\langle D\rangle$. Afterwards the crystal grains are 'build' simulating a simple isotropic $2 \mathrm{D}$ growth which starts simultaneously at all growth centers and is terminated at the locations where the adjacent grains touch each other. The routine stops as soon as the whole element area is covered by the grains. Finally a random direction of the anisotropy axes is assigned to each grain, which means that (i) for all discretization cells within a given grain the anisotropy axes directions are the same, but (ii) the corresponding directions for two different grains are not correlated.

Thin Co films may have a polycrystalline structure with grains having either $f c c$ or $h c p$ crystal lattice type. Co films with thicknesses about several nanometers epitaxially grown on $\mathrm{Cu}$ substrates possess the $f c c$ crystallographic structure (see, e.g., review ${ }^{37}$ and references therein), so it is likely that Co films sputtered on the same substrate (as those used in Ref. 14) also have predominantly the $f c c$ structure (see also results and discussion in Ref. 38). Hence we begin this subsection with the analysis of simulation results for the corresponding case.

\section{Magnetization dynamics for a nanoelement with fcc crystal grains}

At room temperatures the $f c c$ crystallographic modification of Co is unstable for bulk crystals, so that measurements of the anisotropy constant can be performed only on thin films which $f c c$ structure is stabilized by, e.g., a suitable substrate choice (like $\mathrm{Cu}$ with the 111-orientation of the substrate surface). This circumstance leads to considerable difficulties by the anisotropy constant determination, which could be performed mainly on epitaxially grown films (see, e.g., Ref. 39). The values of the first cubic anisotropy constant obtained for thin Co films grown at different conditions on different substrates are within the range $K_{1}^{\mathrm{cub}}=(5-8) \cdot 10^{5} \mathrm{erg} / \mathrm{cm}^{3}$, so we have used the value $K_{1}^{\text {cub }}=6.0 \cdot 10^{5}$ $\mathrm{erg} / \mathrm{cm}^{3}$ in our simulations. Varying $K_{1}^{\mathrm{cub}}$ within the region cited above did not lead to any significant changes of the results. 
From this anisotropy value and the standard saturation magnetization of Co $M_{S} \approx 1400 \mathrm{G}$ (which is almost the same for $f c c$ and $h c p$ phases) one can determine the reduced anisotropy constant $\beta=2 K_{1} / M_{S}^{2}$, also sometimes referred to as a 'quality factor' $Q$. The corresponding value $\beta \approx 0.6$ is not small (and is even larger if we substitute into the expression for $\beta$ the reduced $M_{S}$ value reported in Ref. 14), which means that this random anisotropy may cause substantial inhomogeneities of magnetization structure in a polycrystalline sample. In nanocrystalline thin films, however, where the grain size is of the same order of magnitude as the exchange length, the influence of the random anisotropy is strongly diminished, because its effect is 'averaged out' due to uncorrelated anisotropy directions in adjacent crystallites 40 .

Nevertheless in the nanoelement under study (with the average grain size $\langle D\rangle=10 \mathrm{~nm}$ ) the random cubic anisotropy leads to a noticeable deviations of the magnetization structure from the configuration obtained for an ideal nanoelement (without the polycrystalline structure) and thus results in an observable spread of resonance fields for different crystallites. Qualitatively this leads to the same effect as the decrease of the exchange constant: after passing the threshold value $a_{J}^{\text {cr }}=0.337(2)$ the amplitude of the SPC-driven precession of the average magnetization increases much slower, than in the absence of the random anisotropy. In particular, for $a_{J}=0.340$ we can still observe a really small-angle homogeneous precession with the frequency $f_{\text {hom }} \approx 13.6 \mathrm{GHz}$ which is virtually equal to $f_{\mathrm{FMR}}^{(0)}(\approx 13.7$ $\mathrm{GHz}$ ) - see Fig. 40.

In contrast to the decrease of the exchange constant, which leads to systematic deviations of the magnetization configuration from a homogeneously magnetized state under the influence of the self-demagnetizing field, a crystal grain anisotropy leads to random deviations of the magnetization. For this reason the mode structure after the transition to the quasichaotic precession (Fig. 4: looks entirely different when compared with the case of the reduced exchange constant (Fig. 3. $)$. And finally, the analysis of the oscillation spectra in the quasichaotic region of currents for the nanoelement with a random polycrystalline structure (Fig. 5 - shows that the presence of such an anisotropy slightly expands the existence region of quasichaotic oscillations (compare with Fig. 5 $\mathrm{k}$ ) and significantly decreases the spectral power of 'out-of-plane' oscillations. The latter effect is due to the same reason as for the case of a reduced exchange - random anisotropy disturbs the homogeneous magnetization configuration required for the existence of an 'out-of-plane' precession.

\section{Magnetization dynamics for a nanoelement with hcp crystal grains}

It has been shown experimentally 38 that although crystallites of thin Co films sputtered on $\mathrm{Cu} / 111$ substrates have mostly the $f c c$ structure, there exist a non-negligible fraction of grains possessing a 'normal' $h c p$ structure of bulk Co crystals. For this reason we have also studied the magnetization dynamics of a polycrystalline nanoelement with $h c p$-grains having the same average size $\langle D\rangle=10 \mathrm{~nm}$.

Magnetic anisotropy of the $h c p$ modification of Co can be treated for our purposes as a uniaxial one with only the first anisotropy constant $K_{1}^{\mathrm{cub}}=4.5 \cdot 10^{6} \mathrm{erg} / \mathrm{cm}^{3}$ (see, e.g., Ref. 41) taken into account. The reduced anisotropy constant $\beta \approx 4.6$ calculated from this $K_{1}$ value and the same saturation magnetization $M_{S} \approx 1400 \mathrm{G}$ is so large that the random anisotropy plays a crucial role even in a sample with such small crystallites. This can be seen very well in Fig. 6] where we show maps of the oscillation power for polycrystalline nanoelements with $h c p$ grains. The four maps (a)-(d) were computed for 'samples' with identical macroscopic parameters ('reference' parameter set and one and the same value of the random uniaxial anisotropy of crystallites $\left.K_{1}^{\text {cub }}=4.5 \cdot 10^{6} \mathrm{erg} / \mathrm{cm}^{3}\right)$, but using different realizations of a random polycrystalline structure. The most striking feature of the spectra shown in Fig. [6 is that they are qualitatively different for different 'samples'. In particular, we can recognize both (i) a large spread of the regular oscillation frequencies (narrow peaks for small $a_{J}$ lie in a wide range $\approx 10-20 \mathrm{GHz}$ ) and (ii) very different width and $a_{J}$-dependencies of broad spectral bands in the region of a quasichaotic behaviour. In addition, we observe large frequency jumps by the transition from a regular to a quasichaotic regime (see, e.g., realizations (a) and (b)). This is in strong contrast to the behaviour of a nanoelement with an absent or moderate anisotropy, where the regular oscillation frequency before the transition to a quasichaotic regime was 'inherited' by a main spectral peak of quasichaotic oscillations just after the transition.

Further insight into the magnetization dynamics of a polycrystalline nanoelement with hcp grains can be gained by the analysis of the magnetization trajectories and spatial distribution of the oscillation power. 3D trajectories of the average system magnetization for the 'sample' marked as (b) in Fig. [6 at the $a_{J}$ values before $\left(a_{J}=0.50\right)$ and immediately after $\left(a_{J}=0.54\right)$ the transition from a regular to a quasichaotic regime are shown in Fig. 7 together with $m_{x}^{\text {av }}$ and $m_{z}^{\text {av }}$ power spectra and spatial maps of $m_{x}^{\text {av }}$ and $m_{z}^{\text {av }}$ oscillation power.

First of all we note that due to a strong influence of the $h c p$ anisotropy with randomly oriented anisotropy axes in various grains, the average nanoelement magnetization does not precess anymore approximately around the $0 x$ axis (long ellipse axis) even in the regular regime. This is clearly demonstrated in Fig. 7h: the limiting cycle in the form of a narrow bent torus is strongly tilted with respect to the $0 x$-axis. Such an asymmetric trajectory lead 
to the appearance of a strong spectral peak at the basic precession frequency also in the oscillation spectrum of the longitudinal magnetization component $m_{x}^{\text {av }}$ (compare with spectral maps for an element without a random anisotropy in Fig. 2,

The next important feature is a strongly irregular spatial distribution of the oscillation power which is especially pronounced for the $m_{x}^{\text {av }}$-component (grey-scale map in Fig. 7b). It is evident that this power distribution is determined by the random polycrystalline structure; this circumstance is again in a strong contrast to the case of an absent or moderate $f c c$ anisotropy. By the transition to a quasichaotic regime this spatial distribution changes drastically (compare corresponding maps in Fig. 7b and (7) thus causing a relatively large frequency jump by this transition. For example, the difference between the positions of the peaks corresponding to basic oscillation frequencies in Fig. $7 \mathrm{~b}$ and $7 \mathrm{~b}$ is $\approx 3.5 \mathrm{GHz}$.

Further discussion of these results is again postponed till the next Section.

\section{Magnetization dynamics for finite temperature: influence of thermal fluctuations}

Simulations of the magnetization dynamics taking into account thermal fluctuations were performed by adding the 'fluctuation field' $\mathbf{H}^{\mathrm{fl}}$ to the total effective field in the LLG equation of motion. We have used components of $\mathbf{H}^{\mathrm{fl}}$ with zero means and $\delta$-correlated in space and time

$$
\left\langle H_{\psi, i}^{\mathrm{fl}}(0) H_{\xi, j}^{\mathrm{fl}}(t)\right\rangle=2 D \delta_{\psi, \xi} \delta_{i, j} \delta(t)
$$

where the noise power $D=\left(\lambda / 1+\lambda^{2}\right) \cdot(k T / \gamma \mu)$ depends on the system temperature $T$, damping $\lambda$ and the total magnetic moment of a discretization cell $\mu=M_{S} \Delta V$. In principle it is known that the non-trivial correlation properties of the random noise appear in numerical micromagnetics due to the discretization of a formally continuous problem. We refer the interested reader to Ref. 2742 for the detailed discussion about these properties and their possible influence on the magnetization dynamics. Here we only note that we have checked the issues mentioned in Ref. 27 42 to ensure that the approximation (3) is sufficiently good for our situation.

Another problem arising by numerical simulations at finite temperatures is whether one should change the macroscopic magnetic parameters of the material under study when doing such simulations (see Ref. 43 and citations therein for the recent discussion). Taking into account that we use fairly small discretization cells and simulate the system behaviour at room temperature $(T=300 \mathrm{~K})$ which is well below the Curie temperature of common magnetic materials, we have left the macroscopic parameters unchanged. However, we note that the arguments presented in Ref. 43 make further studies in this direction worthwhile.

We also point out that the inclusion of the fluctuation field $\mathbf{H}^{\mathrm{fl}}$ with correlation properties given by (3) converts the ordinary differential LLG-equation into a stochastic one. Due to random functions appearing on the right-hand side of such equations numerical methods for their solution have, in general, a much lower accuracy order than their counterparts for ordinary differential equations ${ }^{44}$. For this reason a much smaller time step is required to integrate the LLG-equation with the prescribed accuracy at $T>0$. In our particular case, for $T=300 \mathrm{~K}$ and discretization cells with a relatively small size (note that the noise power in Eq. (3) is inversely proportional to the cell volume), the time step had to be decreased by more than order of magnitude. Hence for $T=300 \mathrm{~K}$ we have performed simulations only during the physical time of $\approx 50 \mathrm{~ns}$ (after establishing the steady state oscillation regime). This circumstance explains why the statistical errors in spectra shown in Fig. 8 are considerably larger than for those analysed in previous subsections (for $T=0$ ), especially for a quasichaotic oscillation regime.

Example results of our simulations including thermal fluctuations are displayed in Fig. 8 where we present the analysis of the magnetization dynamics of a polycrystalline nanoelement with a 'reference' parameter set and $f c c$ Co grains.

Comparing these data with the results shown in Fig. 4 and 5 se can see, first of all, that thermal fluctuations have a qualitative influence on the regular precession regime (the region of regular oscillations is marked also in Fig. 8). On the one hand, the $a_{J}$-region where corresponding oscillations exist, is much broader than for $T=0$ : already for $a_{J}=0.2$ the $m_{z}^{\text {av }}$ oscillation power is twice as large as in the absence of a spin current. On the other hand, the width of corresponding spectral lines drastically increases. For $T=0$ the linewidth of $m_{z}^{\text {av }}$-oscillation spectra for a regular precession was always $\Delta f<20 \mathrm{MHz}$, whereby this number represents an upper limit posed on $\Delta f$ by the finite duration of our simulations. Inclusion of a thermal noise leads to strong $a_{J}$-dependent broadening of the spectral lines: at room temperature in the regular regime $\Delta f$ decreases with $a_{J}$ starting from the value $\Delta f \approx 1.5( \pm 0.2) \mathrm{GHz}$ for the spectrum of spontaneous oscillations (without a spin current, $\left.a_{J}=0\right)$ down to $\Delta f \approx 0.4( \pm 0.1) \mathrm{GHz}$ for $a_{J}=0.3$.

The second effect of the thermal noise is the strong decrease of the 'out-of-plane' oscillation power. Comparison of Fig. $8 \mathrm{~b}$ and 5 r shows that in the presence of thermal fluctuations this power is smaller by more than one order 
of magnitude. The reason is the same as explained above by the comparison of results for a 'minimal model' and its modifications presented in Fig. [5]

And finally we note that according to our simulations, thermal noise does not change the frequencies of spectral peaks in a region where a quasichaotic precession regime is well established (starting from $a_{J} \approx 0.38$ for the system studied here). Comparison of corresponding oscillation spectra reveals that the frequencies of both peaks remains the same in frames of statistical errors.

\section{DISCUSSION}

\section{A. Comparison with numerical simulation results of other groups}

Full-scale micromagnetic simulations of a magnetization dynamics driven by a spin-polarized current have been performed, up to our knowledge, only in a few papers, which are partially mentioned in the Introduction.

The first systematic study of the SPC-driven magnetization dynamics was performed in Ref. 28 where a noise induced in a double-layer system with the geometry corresponding to a CPP spin valve head was investigated. Several features of the spin-current induced precession observed in Ref. 28 (fast increase of the magnetization oscillation power after the appearance of a steady state precession, linear dependence of the current threshold value on the damping constant $\lambda$, uniform magnetization precession for low current values and chaotic magnetization motion for large currents, significant low-frequency contribution to the oscillation power for large currents) are qualitatively similar to our results. However, a quantitative comparison is not possible, because geometry and material parameters used in Ref. 28 are very different from ours.

The steady-state magnetization precession in an elliptical single-layer nanoelement 'made of' Permalloy $\left(\mathrm{Ni}_{81} \mathrm{Fe}_{19}\right)$ was studied for the first time in the short report ${ }^{29}$. A polycrystalline structure of a nanoelement was neglected taking into account an extremely small anisotropy of Py grains. Both regular and quasichaotic precession regimes were found in Ref. 29 for an element with lateral sizes $100 \times 50 \mathrm{~nm}$. The increase of the oscillation amplitude beyound the threshold of the steady-state precession onset observed in Ref. 29 is somewhat slower than in our simulations. The current region (normalized on the precession onset value) where a regular precession regime exists in Ref. 29 is broader than for our nanoelements without a polycrystalline structure. Both quantitative discrepancies can be explained by the smaller exchange constant $A=1 \times 10^{-6}$ of Permalloy (compared to our system) and a smaller element size used in Ref. 29.

Simulations most closely related to our studies were done in Ref. 30, where the magnetization dynamics of a single-layer elliptical element with the same geometry as used by us was performed. Lee et al ${ }^{30}$ did not study how the magnetisation dynamics depends on the nanoelement magnetic parameters and did not carry out a systematic analysis of the oscillation spectra, but it is nevertheless possible to compare some results from Ref. 30 with our findings.

First of all we note that Lee et al. simulated a nanoelement with the standard saturation magnetization value of the bulk Co $M_{S}=1400 \mathrm{G}$. Due to this $M_{S}$-value they could obtain a satisfactory agreement between the oscillation frequency in a regular precession regime (called a "small-amplitude signal" in Ref. 30 and 14) obtained in their simulations and experimentally $\stackrel{14}{\underline{1}}$. For example, for the external field $H_{0}=2 \mathrm{kOe}$ simulations performed in Ref. 30 produce $f_{\mathrm{sim}} \approx 16.5 \mathrm{GHz}$ (see Fig. 3b in Ref. 30 ), whereby experiment of the Cornell group gives $14 f_{\exp } \approx 16 \mathrm{GHz}$. This agreement is a natural consequence of a higher $M_{S}$ value used in in Ref. 30 compared to our simulations and the $M_{S}$-value measured for a sample used in Ref. 14: the Kittel FMR-frequency obtained for a thin film with $M_{S}=1400$ $\mathrm{G}$ at $H_{0}=2000 \mathrm{Oe}$ is $f_{\mathrm{FMR}}^{(0)} \approx 17.5 \mathrm{GHz}$. An slightly smaller value simulated in Ref. 30 is most probably due a slightly non-linear precession character (see our discussion of results presented in our Fig. 11).

We have also performed simulations with $M_{S}=1400 \mathrm{G}$ (results are not shown here) and have obtained for a nanoelement with the same geometry a regular precession with the frequency $f_{\text {sim }} \approx 17.2 \mathrm{GHz}$, which is very close both to the value obtained in Ref. 30 and that calculated from the Kittel formula. However, the quasichaotic regime for an element with such a high saturation magnetization starts by our simulations at the frequency $\approx 16 \mathrm{GHz}$ what is approximately 3 times larger than the experimentally observed value (see Fig. 1c and 1f in Ref. 14). In this connection we note, that although the frequencies for a quasichaotic regime are not given in Ref. 30 , the transition scenario from a regular to a quasichaotic precession shown in Fig. 3c from Ref. 30 is similar to ours (see, e.g., Fig. $\square$ in this paper), so that we expect that the frequencies at the beginning of a quasichaotic motion for simulations performed in Ref. 30 are also approximately as high as those for regular oscillations.

In addition, the relation of the maximal to the minimal current of the range where the quasichaotic regime exists for a nanoelement with $M_{S}=1400 \mathrm{G}$ is also much larger than the value $I_{\max } / I_{\min } \approx 3$ deduced from the data presented in Fig. 1f of Ref. 14. The relation $I_{\max } / I_{\min }$ increases, because the orientation (on average) of the magnetization along the external field is preserved up to higher current values for a nanoelement with larger $M_{S}$. Summarizing, the 
usage of a larger saturation magnetization changes the overall agreement between the experiment and simulation for the worse.

It is also instructive to compare our results with the simulations performed in the macrospin approximation in Ref. 14 itself. First of all we note that by performing such simulations one has to introduce the artificial anisotropy field $\mathbf{H}_{\mathrm{an}}$ (directed in this case along the long axis of the ellipsoidal element) in order to reproduce already the quasistatic hysteresis loop. The magnitude of this field (or the corresponding anisotropy constant) is usually chosen to obtain the coercivity similar to those measured experimentally. From the physical point of view this field is intended to describe the average magnetocrystalline anisotropy of a polycrystalline element and/or its shape anisotropy. The first contribution is negligibly small in samples without a specially induced in-plane grain texture (what is probably absent for sputtered Co films). The second contribution - the shape anisotropy of a thin elliptical nanoelement - can not be described adequately by a uniaxial anisotropy term alone, but is in fact a superposition of a strong 'easy plane' anisotropy due to the demagnetizing field of a thin film and an additional in-plane anisotropy arising due to a noncircular lateral shape of a nanoelement. This is one of the reasons why one can expect only a qualitative information from such a macrospin approximation, even if no domain formation is expected during the magnetization reversal.

From this qualitative point of view macrospin simulations presented in Ref. 14 demonstrate two interesting features (see Fig. 3 in Ref. 14): (i) a frequency jump by the transition from a small-angle to a large-angle precession and (ii) an out-of-plane precession mode with the frequency increasing with the current strength.

The first feature is temptingly similar to the frequency jump found for the sample 1 from Ref. 14 when the current was increased (see Fig. 1d and 1f) and hence was proposed by Kiselev et al. as a possible explanation for this experimental observation. Our micromagnetic simulations reveal, however, that in a full micromagnetic model this jump does not occur by the transition from a regular to a quasichaotic precession regime. The reason for this discrepancy between the macrospin and full micromagnetic model is the following. In the macrospin approximation the abrupt transition between the small-angle (ellipsoidal) and a large angle ('butterfly' or 'shell') precession is accompanied by the strong decrease of the oscillation frequency because the length of the 'butterfly' trajectory after the transition is much larger than the length of the elliptical trajectory before it. Full-scale micromagnetic simulations reveal that the regular precession regime exists really up to nearly maximal amplitudes of the magnetization oscillations. This large amplitudes can not be significantly increased by the quasichaotic regime and thus the precession in this regime 'inherits' the frequency of regular oscillations, and the frequency jump by this transition is absent. Hence the abovementioned transition can not be the reason for the frequency jump observed in a real system.

The second feature obtained in the macrospin approximation - the 'out-of-plane' precession regime - is not observed experimentally ${ }^{14}$. Indeed, our full-scale simulations show that this precession mode is relatively unstable due to a slightly non-collinear magnetization configuration already for the 'minimal' model, where neither the polycrystalline structure nor thermal fluctuations are taken into account. Either of these two factors disturbs further the homogeneous magnetization structure, thus nearly wiping out the 'out-of-plane' mode, which intensity is then probably below the observable threshold.

\section{B. Comparison with experimental data}

As it was mentioned in the Introduction, the quality of experimental data presented in Ref. 14 is sufficiently high to allow a quantitative theoretical analysis, but one should of course begin with a qualitative comparison between experimental and simulated results.

First we note that the presence of several spectral bands shown in Fig. 1f in Ref. 14 is due to the superposition of signals arising from the oscillations of the perpendicular and longitudinal magnetization components. This can be clearly seen from Eq. (2) and Fig. 25, where the magnetoresistance oscillation spectrum obtained using (2) is presented. Oscillations of the longitudinal $\left(m_{z}^{\text {av }}\right)$ magnetization component produce the signal with the basic (precession) frequency, whereby the magnetization component perpendicular to the long axis of the ellipsoid $\left(m_{x}^{\text {av }}\right)$ is responsible for the second harmonic generation: As explained above by the analysis of the 'minimal model' spectra (section (IIA), for this type of motion the $m_{x}^{\text {av }}$-component oscillates with the frequency roughly twice the precession frequency. A weak third harmonics is present in the spectrum of $m_{z}^{\text {av }}$-oscillations due to a non-linear precession character for this regime. A quantitative comparison of the basic frequency and harmonic intensities is not possible, because the relation between corresponding intensities strongly depends on the angle between the external field and the ellipsoid axis, which is not known exactly.

The interpretation outlined above is strongly supported by the experimental observation that the basic frequency band disappeared when the external field was aligned as exact as possible along the long axis of the elliptical element 45 , as it should be according to Eq. (2) with $e_{z}^{h}=0$.

The next intriguing feature of the magnetization dynamics measured in Ref. 14 is the existence of two oscillation regimes - for low (for $2.0-2.4 \mathrm{~mA}$ ) and high (from $\approx 2.6 \mathrm{~mA}$ up to $\approx 6.5 \mathrm{~mA}$ ) current values. The transition between 
these two regimes is accompanied by the drastic increase of the microwave power and by the frequency jump from $\approx 16 \mathrm{GHz}$ in a low-amplitude regime down to $\approx 7 \mathrm{GHz}$ for large-amplitude oscillations. These features are quite similar to those obtained for the macrospin model (see corresponding simulations in Ref. 14). However, as explained above, they disappear when this transition is studied using a full micromagnetic model with moderate or absent random grain anisotropy of a polycrystalline elliptical element: First, the frequency during this transition remains nearly unchanged, and second, the magnitude of the simulated microwave power before the transition is at least as high (and for $T=0$ even higher) as immediately after it (this latter feature is also due to a fact that the amplitude in a regular regime just before the transition is by no means small, as it is the case in the macrospin model).

For this reasons another explanation of the frequency and amplitude relation in these two regimes is required. Such an explanation can be given assuming that the nanoelement studied in Ref. 14 had at least a small (but non-negligible) fraction of $h c p$ Co grains (evidences about the coexistence of $f c c$ and $h c p$ phases in this Co films can be found, e.g., in Ref. 38). As shown in Fig. 6] polycrystalline 'samples' consisting of hcp Co demonstrate a large variety of possible spectral maps which strongly depend on the realization of a polycrystalline sample structure. As explained in section IIIC2 this is a natural consequence of a relatively strong anisotropy of the $h c p$ grains, which lead to very different magnetization dynamics for garins with different directions of the anisotropy axes. This, in turn, may lead to a spatial 'migration' of the oscillation power by increasing the current strength. All these circumstances can result in a single (Fig. 6b) or multiply (Fig. 6a) frequency jumps when increasing current and quite different dependencies of the spectral maxima frequency on the current strength (compare the behaviour of broad spectral bands in, e.g., Fig. 6 ; and $\mathrm{d}$ ). The magnitude of the frequency jump can be quite large: we have observed frequency changes as large as 10 $\mathrm{GHz}$ (in principle even larger jumps are possible: the maximal oscillation frequency for a $h c p$ Co grain with $M_{S}$ of a bulk Co and the anisotropy axis aligned along the external field is $f_{\max } \approx 38 \mathrm{GHz}$ ). In this connection it is interesting to note that the regular precession frequency $f_{\text {reg }} \approx 17 \mathrm{GHz}$ obtained for the sample which spectral map is shown in Fig. 6b nearly coincides with that measured in the 'small-amplitude' regime in Ref. 14.

The interpretation proposed above could also explain why the magnitude of the microwave power drastically increases after the transition to a quasichaotic behaviour: it might happen that only a small fraction of a sample (e.g., only $h c p$ grains with a favourable orientation of the anisotropy axes) oscillates in a regular ('small-amplitude') regime, whereby in a quasichaotic regime one observes the oscillations of a major part of a nanoelement occupied by the fcc nanocrystals. Our hypothesis is supported further by the observation that the 'small-amplitude' regime was found not for all experimentally studied samples 45 .

We would like also to add a few comments concerning the 'out-of-plane' precession mode which was not observed experimentally, despite being predicted by a macrospin model. As discussed in subsections IIC 1 IID and IV A above, the absence of this mode in the measured spectra can be explained by the joint influence of a polycrystalline sample structure and thermal fluctuations: both these factors destroy the homogeneous magnetization structure thus decreasing the amplitude of the 'out-of-plane' mode. In this connection it is worth noting that for some $h c p$-'samples' we have observed a relatively weak spectral band corresponding to 'out-of-plane' oscillations (this mode could be identified due to its very characteristic frequency dependence on the current strength similar to that shown in Fig. $3 \mathrm{~b}$ in Ref. 14). We have found out that this mode was localized in a small region of a nanoelement, where the orientations of the grain anisotropy axes were nearly parallel, thus favouring the collinear magnetization structure during the precession. The importance of the polycrystalline structure for the suppression of the 'out-of-plane' precession could be tested on samples made of materials with low crystal grain anisotropy (like Permalloy). For this samples the 'out-of-plane' mode should be observable, because the nearly absent magnetic anisotropy of Py could not destroy the homogeneous magnetization configuration required for the existence of the 'out-of-plane' mode. This conclusion seems to be supported by the recent experimental observations ${ }^{16}$.

As it can be seen from the previous discussion, the extended micromagnetic model which takes into account a polycrystalline structure of a nanoelement and thermal fluctuations can explain qualitatively all most important features of the experimental data reported up to now ${ }^{14}$. However, from the quantitative point of view, several discrepancies do exist.

The most important one is the difference between the simulated and measured frequencies in the quasichaotic regime: at the beginning of this regime the measured frequency is $f_{\text {mes }} \approx 6.5 \mathrm{GHz}$ (see Fig. 1c in Ref. 14), whereas our simulations for elements without a polycrystalline structure and for a polycrystalline $f c c$-Co give $f_{\text {sim }} \approx 9-10$ $\mathrm{GHz}$ for $a_{J}$ values where the transition to a quasichaotic regime is completed (see, e.g., Fig. 11 and spectrum for $a_{J}=0.38$ in Fig. 8). We consider this discrepancy as the most serious one because the frequency is the most reliable spectral characteristic obtained both in measurements and simulations: whereas other parameters like the line width and intensity can be artificially altered by many factors, the peak frequency is an inherent signal feature which is usually reproduced with an accuracy allowing a direct comparison between simulations and experiment.

By the discussion of the influence of various factors which could account for the frequency difference pointed out above we would like to begin with the reasons having no direct relation to the spin injection, i.e., with the factors which are not taken into account in our 'standard' micromagnetic model. 
(i) Influence of the underlayer. In our treatment we have considered a single-layer elliptical nanoelement, which means that the magnetodipolar interaction with the Co underlayer present in a real experiment 14 was not included. In principle, such an interaction could indeed decrease the oscillation frequency, especially taking into account that the underlayer could be also partially structured by the ion milling used in Ref. 14 to produce an elliptical nanoelement from the upper Co layer. The elliptical pillar sticking out from the underlayer after such a milling would be magnetized in the external field direction thus producing the stray field in the opposite direction. This latter field would decrease (on average) the total field acting on the elliptical nanoelement under study thus decreasing its precession frequency.

We are not able to simulate this effect quantitatively because the milling depth for the underlayer is not known exactly. However, by creating the columnar structure studied in Ref. 14 it was aimed to disturb the lower Co layer as little as possible, so that the milling depth through the underlayer should be maximal several nanometers 45 . The stray field from a pillar with such a small height acting on the element placed $10 \mathrm{~nm}$ above it would be concentrated mainly in small regions near those edges of the nanoelement which are perpendicular to the external field (or the long ellipsoid axis). Thus such a field could presumably not lead to a significant decrease of the precession frequency in the quasichaotic regime, where oscillations of the inner nanoelement regions play a major role.

To check this conclusion, we have performed simulations of a system consisting of an extended underlayer with a thickness $10 \mathrm{~nm}$, an elliptical pillar with the thickness $5 \mathrm{~nm}$ and lateral sizes $130 \times 70 \mathrm{~nm}$ placed on the top of it and a nanoelement with the same lateral sizes located $10 \mathrm{~nm}$ (thickness of the $\mathrm{Cu}$ layer between the two Co layers in Ref. 14) above the pillar. Periodic in-plane boundary conditions were applied to eliminate the effect of the stray field from a relatively thick extended underlayer. We have found (detailed results will be presented elsewhere) that the precession frequency both in the regular and in the quasichaotic regime was not noticeably influenced by the underlayer with a pillar, as expected according to the considerations above.

(ii) Grain texture for an element with a hcp polycrystalline anisotropy. The already studied influence of the polycrystalline structure for a Co nanoelement with hcp grains could be greatly enhanced if the element would possess a significant grain texture. In particular, if there would exist an element region with the lateral size much larger than the element thickness where the grain anisotropy axes are approximately parallel to each other and perpendicular to the element plane, then the oscillation frequency for a homogeneous precession mode of such a region would be $f_{\mathrm{FMR}}^{\mathrm{an}}=(\gamma / 2 \pi) \cdot\left[H_{0}\left(H_{0}+(4 \pi-\beta) M_{S}\right)\right]^{1 / 2}$ (for $\left.\beta<4 \pi\right)$, where $\beta=2 K / M_{S}^{2}$ is the reduced anisotropy constant introduced above. For a $h c p$ Co with $M_{S}=1400 \mathrm{G}$ and $K=4.5 \times 10^{6} \mathrm{erg} / \mathrm{cm}^{3}$ this means the reduction of the precession frequency by a factor $f_{\mathrm{FMR}}^{(0)} / f_{\mathrm{FMR}}^{\mathrm{an}} \approx 1.2$. If we assume that the quasichaotic precession frequency $f_{\mathrm{ch}}$ scales with $M_{S}$ and $\beta$ at least approximately in the same way as the FMR-frequency, the influence of the $h c p$-anisotropy could decrease $f_{\text {ch }}$ from $10 \mathrm{GHz}$ down to $\approx 8.2 \mathrm{GHz}$ which would significantly improve the agreement with the experiment. For a Co film with the reduced $M_{S} \approx 800 \mathrm{G}$ the anisotropy $\beta \approx 14$ would be even somewhat larger than the shape anisotropy so that the precession with the frequency close to the free spin value $f_{0}=(\gamma / 2 \pi) \cdot H_{0} \approx 5.5 \mathrm{GHz}$ and lower (taking into account that $f_{\mathrm{ch}}<f_{\mathrm{FMR}}^{(0)}$ ) could be expected. Unfortunately, as stated above, a highly textured polycrystalline structure should exist in the sample under study in order to make these arguments applicable. This is unlikely for a sputtered Co film (we note in addition, that such an area would probably lead to an appearance of a significant peak at the corresponding frequency in a regular regime). However, this line of the argumentation clearly shows that a detailed characterization of a polycrystalline sample structure is necessary when one performs measurements on materials with moderate to high magnetocrystalline anisotropy.

(iii) Surface roughness. In a thin film element with a rough surface a demagnetizing field due to this roughness would exist, what can also decrease a precession frequency. Quantitative studies of the roughness effect are possible only when the corresponding information about the roughness parameters is available. Here we can only point out that a substantial roughness with a characteristic wavelength of the same order as the element thickness is necessary to create an appreciable demagnetizing field inside a nanoelement.

Next we consider in our discussion the possible factors connected to the magnetization dynamics of a system driven by a spin-polarized current (SPC).

(iv) Effective field induced by the spin-injection. The existence of such a field was extensively discussed theoretically (see references in the Introduction), but only recently this field could be measured in a direct experiment 46 using a small asymmetry of the hysteresis loop in a multilayer system in the presence of a SPC. For a system very similar to that studied in Ref. 14 it was shown that the magnitude of a SPC-induced effective field can be calculated as $H_{\mathrm{SPC}}=b_{J} \cdot j$, where $j$ is the current density and the coefficient $b_{J}$ was found to be $b_{J} \approx 1.5 \times 10^{-7} \mathrm{Oe} \times \mathrm{cm}^{2} / A$. For a typical current value $I=3 \mathrm{~mA}$ and lateral sizes of the nanoelement under study $130 \times 70 \mathrm{~nm}$ the current density is $j \approx 4 \cdot 10^{7} \mathrm{~A} / \mathrm{cm}^{2}$. Hence a SPC-induced field would be $H_{\mathrm{SPC}} \approx 6$ Oe which is negligibly small.

(v) Non-trivial angular dependence of the Slonczewski torque magnitude $a_{J}$ and configuration-dependent damping $\lambda$. As it was discussed in Sec. III these factors should be considered on equal footing, because the spin-induced torque and precession damping in multilayer systems subject to a spin-polarized current are controlled by the processes of the same physical nature. Here we would like only to mention, that from a qualitative point of view the enhancement of the precession damping for large SPC strengths and/or for large angles between the magnetization of adjacent layers 
(see Ref. in Sec. II) could indeed decrease the precession frequency in a quasichaotic regime which is characterized by strong inhomogeneities of the magnetization structure. The quantitative study of the corresponding effect is the subject of a future work.

\section{CONCLUSION}

In conclusion, we have performed a systematic study of the magnetization dynamics of a thin single-layer elliptical nanoelement driven by a spin-polarized current flowing perpendicular to its plane. Analyzing the dependence of this dynamics on various magnetic material parameters, we have demonstrated that an extended micromagnetic model which takes into account a polycrystalline structure of this element and the influence of thermal fluctuations can in principle explain qualitatively almost all features observed experimentally. At the same time we have shown that an apparent qualitative agreement between the macrospin model and some experimental findings is due to artificial features of the macrospin dynamics, which are absent in the full-scale micromagnetic model. An important issue concerning the quantitative disagreement between the precession frequencies measured experimentally and simulated numerically for the quasichaotic regime still requires further clarification. Analyzing various possible causes of this discrepancy we have shown that the most likely candidate to resolve this difficulty is the refined model of the spininjection induced dynamics which would include the non-trivial angular dependence of the spin-torque term and the dependence of the precession damping on the spin-polarized current strength and the magnetization configuration of a multilayer system.

1 J.C. Slonczewski, J. Magn. Magn. Mat., 159, L1, (1996); L. Berger, Phys. Rev. B, 54, 9353 (1996)

2 J.Z. Sun, J. Magn. Magn. Mat., 202, 157 (1999); M. Tsoi, A.G.M. Jansen1, J. Bass1, W.-C. Chiang, M. Seck, V. Tsoi, P. Wyder, Phys. Rev. Lett., 80, 4281 (1998)

${ }^{3}$ G. Reiss, H. Brueckl, A. Huetten, J. Schmalhorst, M. Justuts, A. Thomas, S. Heitman, phys. stat. sol. (b) 236, 289 (2003);I. Zutic, J. Fabian, S. Das Sarma Rev. Mod. Phys. 76, 323 (2004); G. Schmidt, J. Phys. D: Appl. Phys. 38, R107 (2005)

4 X. Waintal, E.B. Myers, P.W. Brouwer, D.C. Ralph, Phys. Rev. B, 6212317 (2000)

5 M.D.Stiles, A.Zangwill, Phys. Rev. B, 66014407 (2002)

6 M.D.Stiles and A.Zangwill, J. Appl. Phys. 916812 (2002)

7 J.C. Slonczewski, J. Magn. Magn. Mat., 247324 (2002)

8 J. Manshot, A. Brataas, G. Bauer, Phys. Rev. B, 69092407 (2004)

9 J. Xiao, A. Zangwill, M.D. Stiles, Phys. Rev. B, 70, 172405 (2004)

10 Y. Tserkovnyak, A. Brataas, G. Bauer, Phys. Rev. Lett., 88117601 (2002)

11 Y. Tserkovnyak, A. Brataas, G. Bauer, Phys. Rev. B, 67140404 (2003)

12 Y. Tserkovnyak, A. Brataas, G. Bauer, J. Appl. Phys., 937534 (2003)

13 B. Heinrich, G. Woltersdorf, R. Urban, E. Simanek, J. Appl. Phys. 93, 7545 (2003)

14 S.I. Kiselev, J.C. Sankey, I.N. Krivorotov, N.C. Emley, R.J. Schoelkopf, R.A. Buhrman, D.C. Ralph, Nature, 425, 380 (2003);

15 S.I. Kiselev, J.C. Sankey, I.N. Krivorotov, N.C. Emley, M. Rinkoski, C. Perez, R.A. Buhrman, D.C. Ralph, Phys. Rev. Lett., 93, 036601 (2004).

16 S.I. Kiselev, J.C. Sankey, I.N. Krivorotov, N.C. Emley, M. Rinkoski, R.A. Buhrman, D.C. Ralph, contributed talk on JMMM04, USA, November 2004, to appear in: J. Appl. Phys.

17 G.D. Fuchs, N.C. Emley, I.N. Krivorotov, P.M. Braganca, E.M. Ryan, S.I. Kiselev, J.C. Sankey, D.C. Ralph, R.A. Buhrman, J. A. Katine, Appl. Phys. Lett., 85, 1205 (2004)

18 M. Covington, M. AlHajDarwish1, Y. Ding2, A. Rebei, G.J. Parker, N. Gokemeijer, M.A. Seigler, J. Magn. Magn. Mat. 287 325 (2005)

19 W.H. Rippard, M.R. Pufall, T.J. Silva, Applied Physics Letters 82, 1260 (2003).

${ }^{20}$ W.H. Rippard, M.R. Pufall, S. Kaka, S.E. Russek, T.J. Silva, Phys. Rev. Lett., 92, 027201-1 (2004)

21 W.H. Rippard, M.R. Pufall, S. Kaka, T.J. Silva, S.E. Russek Phys. Rev. B, 70, 100406(R) (2004)

22 J. Miltat, G. Albuquerque, A. Thiaville, C. Vouille, J. Appl. Phys., 89, 6982 (2001)

23 J.A. Katine, F.J. Albert, R.A. Buhrman, E.B. Myers, D.C. Ralph, Phys. Rev. Lett., 84,3149 (2000); J. Grollier, V. Cros, A. Hamzic, J. M. George, H. Jaffrs, A. Fert, G. Faini, J. Ben Youssef, H. LeGall, Appl. Phys. Lett., 78, 3663 (2001)

24 J.Z. Sun, Phys. Rev. B, 62570 (2000)

25 Z. Li, S. Zhang, Phys. Rev. B, 68 024404-1 (2003)

26 D.V. Berkov, N.L. Gorn, Phys. Rev. B, 71052403 (2005)

27 D.V. Berkov, N.L. Gorn, J. Magn. Magn. Mat., 290-291P1 (2005) 442-448

28 J.-G. Zhu, X. Zhu, IEEE Tarns. Magn., 40, 182 (2004)

29 X. Zhu, J.-G. Zhu, R.M. White, J. Appl. Phys., 95, 6630 (2004) 
${ }^{30}$ K.J. Lee, A. Deac, O. Redon, J.P. Nozieres, B. Dieny, Nature Materials, 3 (2004) 877

31 D.V. Berkov, N.L. Gorn, MicroMagus - package for micromagnetic simulations, http: \\www.micromagus.de

32 E.C. Stoner, Rep. Progr. Phys., 1383 (1950)

33 W. Doering, Micromagnetismus, in: Handbook of Physics, ed. by S. Flgge, Springer-Verlag Berlin, 1966

34 G. Shirane, V.J. Minkiewicz, R. Nathans, J. Appl. Phys., 39, 383 (1968)

35 A. Michels, J. Weissmüller, A. Wiedenmann, J.S. Pedersen, J.G. Barker, Phil. Mag. Lett., 80785 (2000)

${ }^{36}$ G. Bauer, A. Brataas, D. Huertas-Hernando, Y. Tserkovnyak, Phys. Rev. B, 67094421 (2003)

37 K. Heinz, S. Mller, L. Hammer, J. Phys.: Cond. Matt., 11 (1999) 9437

38 J. Langer, R.Mattheis, B.Ocker, W. Mass, S. Senz, D. Hesse, J. Krusslich, J. Appl. Phys., 90, 5126 (2001)

39 D. Weller, G.R.Harp, R.F.C. Farrow, A. Cebollada,J. Sticht, Phys. Rev. Lett., 72 (1994) 2097; R.M. Osgood III, K.T. Riggs, A.E. Johnson, J.E. Mattson, C.H. Sowers, S.D. Bade, Phys. Rev. B, 56 (1997) 2627;

40 G. Herzer, Nanocrystalline Soft Magnetic Alloys, in: Handbook of Magnetic Materials, vol. 10, Ed. by K. Buschov, Elsevier Science, 1997

41 S. Chikazumi, Physics of Ferromagnetism, Clarendon Press, Oxford, 1997

42 D.V. Berkov, N.L. Gorn, J. Magn. Magn. Mat., 272-276P1 (2004) 687

43 G. Grinstein, R.H. Koch, Phys. Rev. Lett., 90 207201-1 (2003)

44 P.E.Kloeden, E.Platen, Numerical Solution of Stochastic Differential Equations, Springer-Verlag, Berlin, 1995

45 S.I. Kiselev, private communication

46 M.A. Zimmler, B. zyilmaz, W. Chen, A.D. Kent, J.Z. Sun, M.J. Rooks, R.H. Koch Phys. Rev. B 70 (2004) 184438 


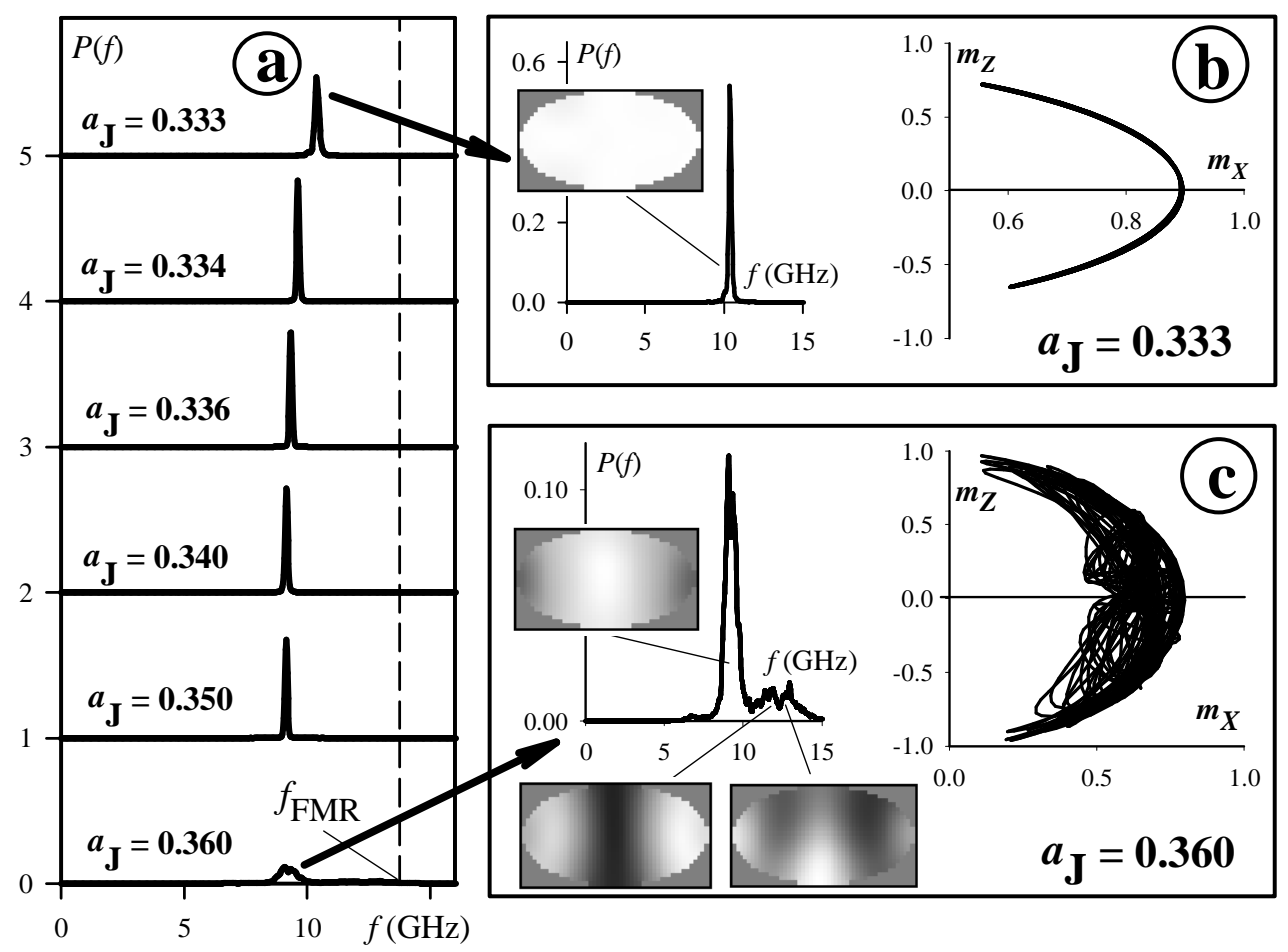

FIG. 1: Magnetization dynamics for an elliptical element with a 'reference' parameter set near the oscillation onset threshold. Panel (a): power spectra of the $m_{z}^{\text {av }}$-component for various $a_{J}$ values as indicated in the figure. Panel (b): magnetization dynamics for $a_{J}=0.333$ represented by the enlarged $m_{z}^{\mathrm{av}}$-oscillation spectrum and the projection of the average magnetization trajectory on the coordinate plane $0 x z$ (coinciding with the nanoelement plane). Panel (c): the same as on (b) for $a_{J}=0.360$ - immediately after the transition to a quasichaotic oscillation regime. Grey-scale maps represent spatial distributions of the $m_{z}^{\text {av }}$ oscillation power for corresponding spectral peaks. 
FIG. 2: (Color on-line) Spectral power of magnetization oscillations for an elliptical element with a 'reference' parameter set for $a_{J}$ values above the transition from a regular to a quasichaotic regime. Oscillation power of $m_{x}^{\text {av }}$ (a) and $m_{z}^{\text {av }}$ (b) magnetization components together with the magnetoresistance oscillation power (c) calculated from the relation (2) are presented. A region on the $\left(a_{J}-f\right)$-plane corresponding to the spectral peaks shown in Fig. 1 (regular precession regime) is marked as a white rectangle in the panel (b) 


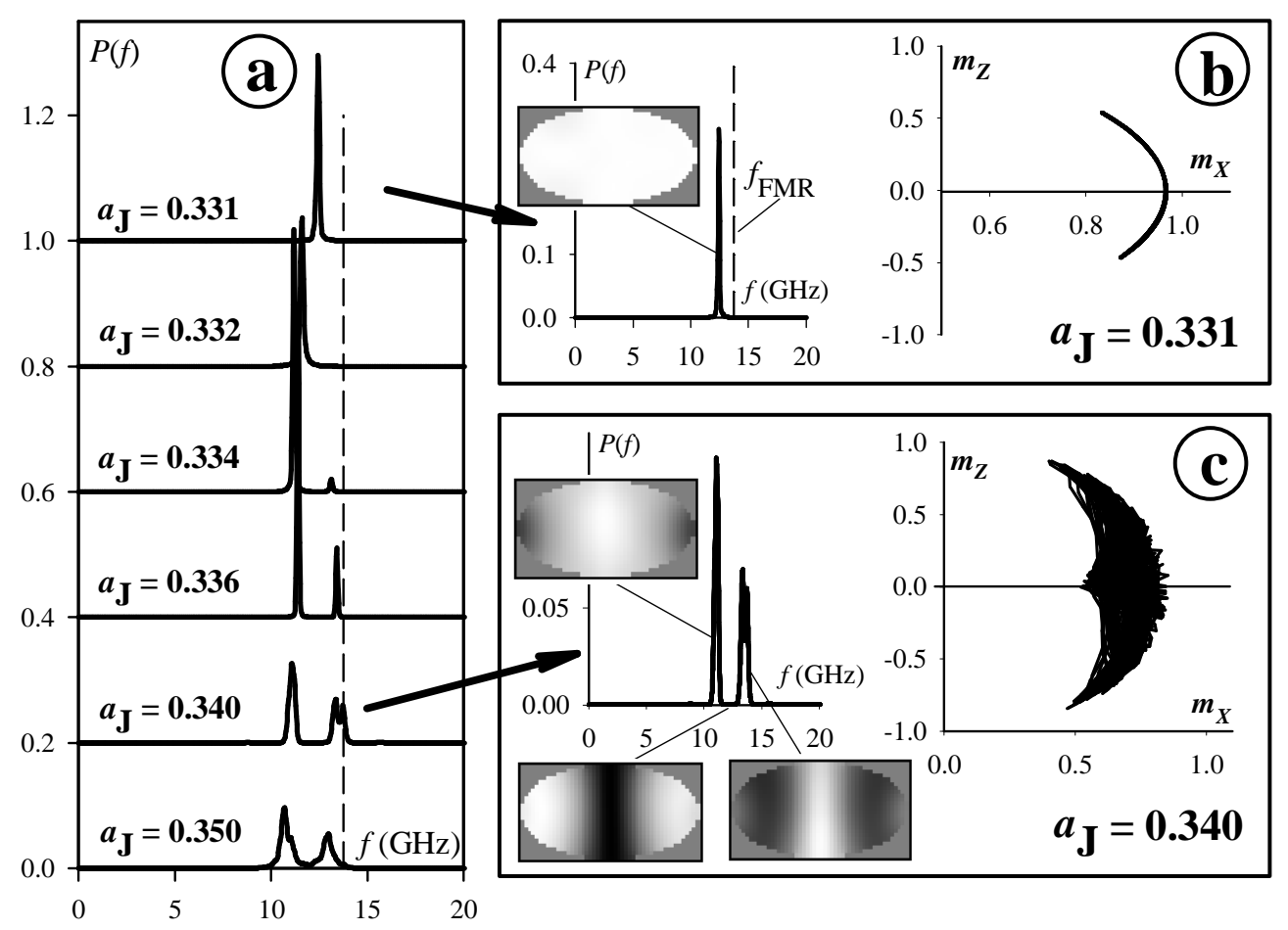

FIG. 3: Magnetization dynamics for a nanoelement with the exchange constant reduced down to $A=2.0 \cdot 10^{-6} \mathrm{erg} / \mathrm{cm}(\mathrm{other}$ parameters are as for a 'reference' parameter set) represented in the same way as in Fig. 1 Note that the maximal oscillation power in a regular precession regime is much smaller than in Fig. 1 


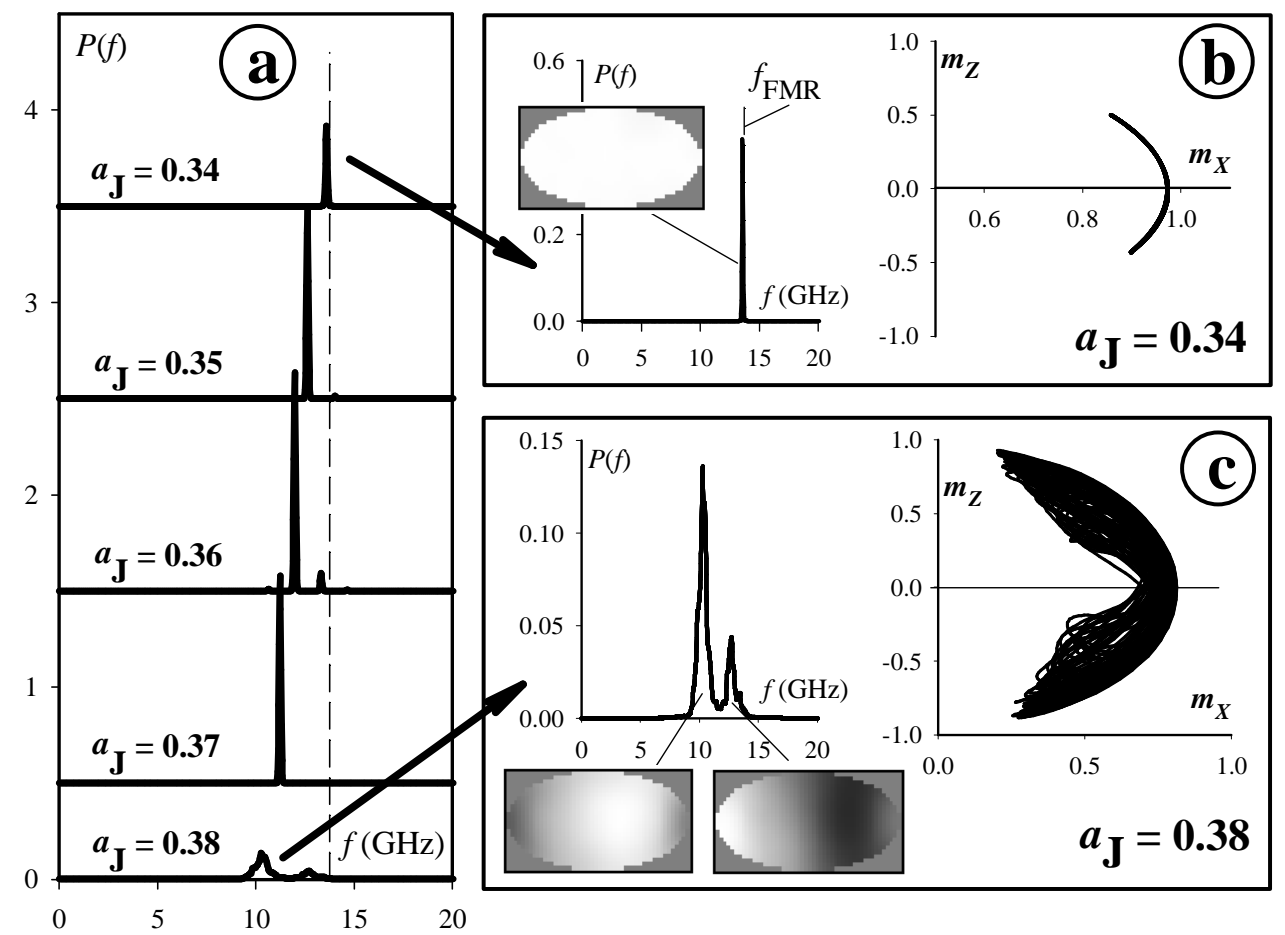

FIG. 4: The same as in Fig. 1 for a nanoelement with the 'reference' parameter set and the random magnetic anisotropy corresponding to a polycrystalline $f c c$ Co film structure with the average grain size $\langle D\rangle=10 \mathrm{~nm}$. 
FIG. 5: (Color on-line) Comparison of the magnetoresistance power spectra in a quasichaotic regime for nanoelements with the 'reference' parameter set (a), the 'reference' parameter set except the reduced exchange stiffness constant $A=2.0 \cdot 10^{-6}$ $\mathrm{erg} / \mathrm{cm}(\mathrm{b})$, the 'reference' parameter set and a polycrystalline structure with average grain size $\langle D\rangle=10 \mathrm{~nm})(\mathrm{c})$. For the last case the random orientations of anisotropy axes for different grains and the $f c c$ Co structure (cubic anisotropy of grains with $K_{1}^{\text {cub }}=6.0 \cdot 10^{5} \mathrm{erg} / \mathrm{cm}^{3}$ ) were assumed. It can be seen that both the reduction of the exchange stiffness constant and inclusion of a random anisotropy lead to (i) the expansion of the $a_{J}$ region where quasichaotic oscillations exist and (ii) decrease of the out-of-plane oscillation power.

FIG. 6: (Color on-line) Maps of the magnetoresistance oscillation power for a nanoelement with the 'reference' parameter set and a polycrystalline structure with $h c p h c p$ Co grains. The grains with $\langle D\rangle=10 \mathrm{~nm}$ have a uniaxial anisotropy $\left(K_{1}^{\text {un }}=4.5 \cdot 10^{6}\right.$ $\mathrm{erg} / \mathrm{cm}^{3}$ ) and randomly oriented anisotropy axes. Maps (a)-(d) represent oscillation power spectra for nanoelements with the same macroscopic parameters, but different realizations of a random polycrystalline structure. 
3D magn. trajectory
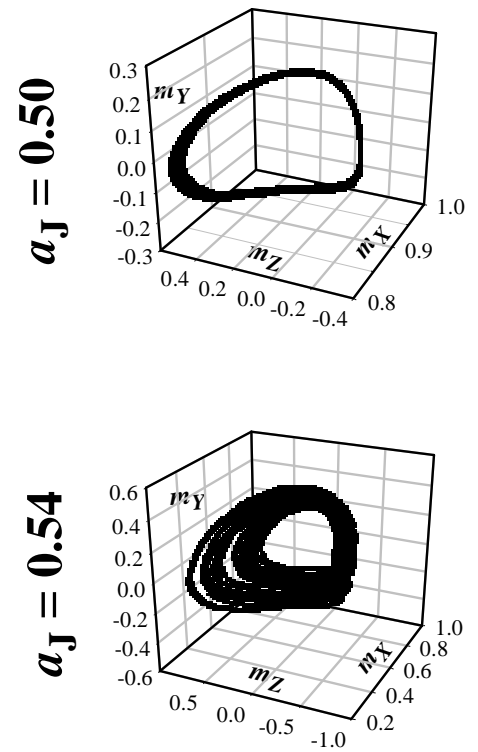

$m_{X}$ osc. power
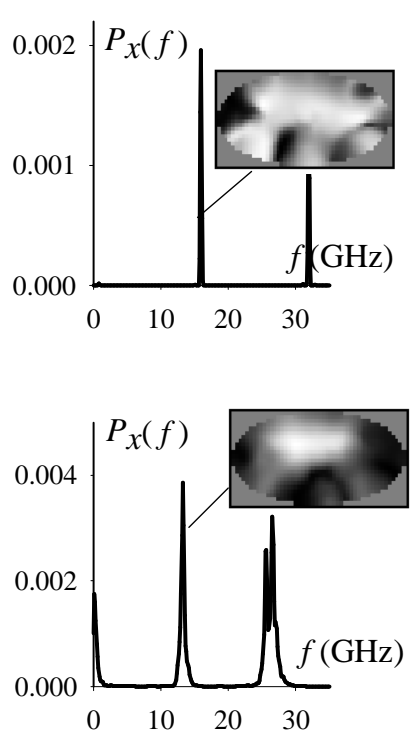

$\boldsymbol{m}_{Z}$ osc. power
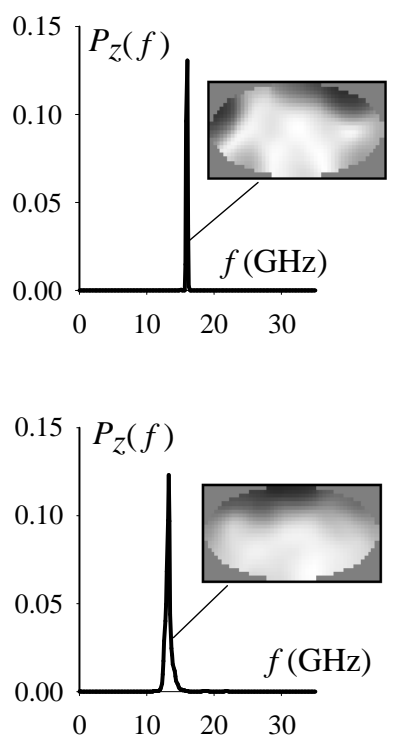

FIG. 7: Magnetization trajectories (a,d) and oscillation power spectra for $m_{x}^{\text {av }}(\mathrm{b}, \mathrm{e})$ and $m_{z}^{\text {av }}(\mathrm{c}, \mathrm{f})$ magnetization components for a random polycrystalline structure realization marked as (b) in Fig. 6 The graphs in the upper row are for the current value $a_{J}=0.50$ before the transition from a regular to a quasichaotic behaviour, the lower row shows results for $a_{J}=0.54$ which is immediately after this transition. Maps in the graphs (b),(c),(e) and (f) display the spatial distribution of the oscillation power for the corresponding magnetization components. 
FIG. 8: (Color on-line) Magnetization dynamics of a polycrystalline ( $f c c$ Co) nanoelement at $T=300 \mathrm{~K}$ : transition from a regular to a quasichaotic behaviour (a) and the map of the magnetoresistance oscillation power for the whole $a_{J}$-range (b). Comparison of the latter map to the analogous map for $T=0$ in Fig. 5 (c) shows that thermal fluctuations lead to (i) an appearance of an extended region of a regular precession and (ii) a strong power decrease of the 'out-of-plane' oscillations. 
This figure "Fig2_Berkov_SpinInj_EllipticalElem.gif" is available in "gif" format fro http://arxiv.org/ps/cond-mat/0503754v2 
This figure "Fig5_Berkov_SpinInj_EllipticalElem.gif" is available in "gif" format fro http://arxiv.org/ps/cond-mat/0503754v2 
This figure "Fig6_Berkov_SpinInj_EllipticalElem.gif" is available in "gif" format fro http://arxiv.org/ps/cond-mat/0503754v2 
This figure "Fig8_Berkov_SpinInj_EllipticalElem.gif" is available in "gif" format fro http://arxiv.org/ps/cond-mat/0503754v2 